\title{
Direct Small Molecule Activation of Mitofusins
}

Emmanouil Zacharioudakis ${ }^{1}$, Nikolaos Biris ${ }^{1}$, Thomas P. Garner ${ }^{1}$, Yun $\mathrm{Chen}^{2}$, Ryan, Pekson ${ }^{2}$, Rimpy Dhingra ${ }^{3}$, Gaetano Santuli ${ }^{4}$, Lorrie A. Kirshenbaum ${ }^{3}$, Richard N. Kitsis ${ }^{5 *}$, Evripidis Gavathiotis $^{6^{*}}$

${ }^{1}$ Department of Biochemistry, Albert Einstein College of Medicine, Bronx, NY 10461 USA

${ }^{2}$ Department of Medicine and Wilf Family Cardiovascular Research Institute, Albert Einstein College of Medicine, Bronx, NY, 10461 USA

${ }^{3}$ Department of Physiology and Pathophysiology and Department of Pharmacology and Therapeutics, Max Rady College of Medicine, Rady Faculty of Health Sciences, University of Manitoba, Winnipeg, Manitoba Canada and Institute of Cardiovascular Sciences, St. Boniface Research Centre, Winnipeg, Manitoba, R2H2A6 Canada

${ }^{4}$ Department of Medicine, Wilf Family Cardiovascular Research Institute, and Einstein-Mount Sinai Diabetes Research Center, Albert Einstein College of Medicine, Bronx, NY, 10461 USA ${ }^{5}$ Department of Medicine, Department of Cell Biology, Wilf Family Cardiovascular Research Institute, Albert Einstein Cancer Center, and Einstein-Mount Sinai Diabetes Research Center, Albert Einstein College of Medicine, Bronx, NY 10461 USA

${ }^{6}$ Department of Biochemistry, Department of Medicine, Wilf Family Cardiovascular Research Institute, and Albert Einstein Cancer Center, Albert Einstein College of Medicine, Bronx, NY 10461 USA

*To whom correspondence should be addressed:

Evripidis Gavathiotis, Ph.D.

Albert Einstein College of Medicine

Forchheimer G46

1300 Morris Park Avenue

Bronx, NY 10461 USA

Tel: 7184303725

Email: evripidis.gavathiotis@einstein.yu.edu

Richard N. Kitsis, MD.

Albert Einstein College of Medicine

Forchheimer G46

1300 Morris Park Avenue

Bronx, NY 10461 USA

Tel: 7184302609

Email: richard.kitsis@einstein.yu.edu 


\section{Summary}

45 Mitochondrial fusion is a physiological process that is regulated by mitofusins on the outer mitochondrial membrane. Conformational plasticity between anti- and pro-tethering

47 conformations of mitofusins permits mitochondrial tethering and subsequent fusion. Here

48 we developed a pharmacophore-based model to rationally manipulate the conformational

49 plasticity of mitofusin 2 and perfomed an in silico small-molecule screen. This enabled the

50 discovery of a direct activator of mitofusins, MASM7, capable of potently promoting

51 mitochondrial fusion. The specificity of the MASM7-mitofusin 2 interaction is highlighted

52 by structure-activity relationships of MASM7 analogues, FRET, NMR and mitochondrial

53 fusion studies using mitofusin mutants. Our study identified the first-in-class direct

54 activator of mitofusins, demonstrating a new paradigm for chemical modulation of

55 mitochondrial fusion and downstream processes.

Keywords: mitochondrial dynamics; mitochondrial fusion, mitofusins; pharmacophore model, in 


\section{Introduction}

68 Mitochondria are highly dynamic organelles that fuse and divide constantly. The physiological

69 processes that regulate this mitochondrial dynamism are fusion and fission, respectively (Detmer

70 et al., 2007). A large variability in mitochondrial morphology is observed among different cell

71 types and within the same cell, as mitochondria can morph into small spheres, short rods or long

72 tubules. This dynamism allows mitochondria to exchange contents (e.g. lipid membranes,

73 proteins), promote repair, and maintain mitochondrial quality control (Shirihai et al., 2015).

The fusion machinery includes mitofusin (Mfn) 1 and 2 on the outer mitochondrial membrane (OMM) and optic atrophy (Opa) 1 on the inner membrane (Shirihai et al., 2015; Schrepfer et al., 2016). Mfn1 and Mfn2 share high sequence homology (Schrepfer et al., 2016). Both homologs possess an N-terminal GTPase domain, a coiled-coiled heptad repeat (HR1) domain, a short transmembrane domain responsible for anchoring Mfns on the OMM, and a second coiled-coiled heptad repeat (HR2) domain located in the C-terminus (Figure 1A) (Shirihai et al., 2015; Schrepfer et al., 2016; Koshiba et al., 2004). The initial step in fusion is tethering of the OMMs of adjacent mitochondria. HR2 domains of Mfns from two adjacent mitochondria

82 interact in an anti-parallel trans manner to form homotypic (Mfn1-Mfn1 or Mfn2-Mfn2) or heterotypic (Mfn1-Mfn2) complexes, subsequently mediating mitochondrial tethering (Koshiba et al., 2004). We recently proposed a model in which Mfn2 can undergo conformational

85 activation to promote mitochondrial tethering (Franco et al., 2016). Based on this model, Mfn2

86 can adopt anti- or pro-tethering conformations (Figure 1A) (Franco et al., 2016). In the anti-

87 tethering conformation HR2 interacts intra-molecularly with HR1 in an anti-parallel manner

88 (Figure 1A). On the other hand, in the pro-tethering conformation HR1-HR2 interactions are

89 disrupted, and the HR2 domain extends into the cytosol to mediate mitochondrial tethering 
90 through anti-parallel HR2-HR2 interactions as proposed by Koshiba et al. (2004) (Figure 1A).

91 Recently, Cao et al. (2017) demonstrated that dimerization of GTPase domains of mitofusins

92 may also control mitochondrial tethering. In contrast, Mattie et al. (2018) proposed an alternative

93 model for mitochondrial tethering, in which Mfns are activated by HR2 domain dimerization in

94 the intermembrane mitochondrial space. Further work will be needed to resolve the differences

95 in these models.

96 Irrespective of the precise tethering model, however, we and others have shown

97 disruption of the HR1-HR2 interaction is critical for mitochondrial tethering and fusion to take

98 place (Franco et al. 2016, Huang et al., 2011). Therefore, we sought to identify mitofusin

99 activating small molecules (MASMs) by targeting the disruption of the HR1-HR2 interaction.

100 To accomplish this, we used the HR1-HR2 interaction that is supported by the structural model

101 of full-length Mfn2 (Franco et al. 2016) and the crystal structures of Mfn1 (Cao et al., 2017).

102 Derangements/imbalances in mitochondrial dynamics can lead to neurological,

103 metabolic, cardiovascular disorders, as well as cancer (Detmer et al., 2007; Shirihai et al., 2015;

104 Schrepfer et al., 2016). For example, impaired mitochondrial fusion in the rare neurological

105 disease Charcot Marie Tooth disease type 2A (CMT2A), can result from a plethora of loss of

106 function mutations in Mfn2 (Bombelli et al., 2014). Indeed, we have shown that the HR1-based

107 peptide, 367-384 Gly and the small molecule described herein can correct the CMT2A Mfn2

108 mutant phenotype (Franco et al., 2016; Rocha et al., 2018). This paper describes the discovery

109 of this small molecule, which may provide a drug prototype for CMT2A as well as for more 110 common acquired disorders mediated by defects in mitochondrial fusion.

\section{RESULTS AND DISCUSSION}


113 To identify small molecules that directly activate Mfn2 and promote mitochondrial fusion, we

114 subjected Mfn2 to a structure-guided pharmacophore-based approach combined with in silico

115 screening with the goal of directly promoting the pro-tethering and/or inhibiting the formation of

116 anti-tethering conformation of Mfn2. Our previous structural model of full-length Mfn2 in the

117 anti-tethering conformation (Franco et al., 2016) and the recent crystal structure of Mfn1 (Cao, et

118 al., 2017) informed us about the intra-molecular HR1-HR2 interactions,. Visual inspection of the

119 intra-molecular HR1-HR2 interactions in the human Mfn2 structural model and particularly in

120 the region that involves HR1-residues: 367-384, which our previous work using a 367-384 Gly

121 peptide suggested to regulate, provided structural insights for small molecule mimicry (Franco et

122 al., 2016). Specifically, several hydrophobic interactions were observed between the following

123 HR1-amino acids: Val372, Met376 and HR2-amino acids: Leu724, Leu727 and Ala731 and a

124 possible hydrogen bond between the HR1-amino acid: His380 and HR2-amino acid: Asp725

125 (Figure 1B). All of these residues are highly conserved in Mfn2 and Mfn1 of different species

126 (Figure S1A). We envisioned that a small molecule capable of recapitulating the interactions of

127 the aforementioned HR1-amino acids would compete with intra-molecular HR1-HR2

128 interactions and promote Mfn2 conformational activation. Accordingly, to identify such a small

129 molecule, we generated a pharmacophore hypothesis that comprises the following features: three

130 hydrophobic interactions, an aromatic interaction and a hydrogen bond donor based on the

131 interactions of the HR1 residues: Val372, Met376 and His380 (Figure 1C). An in silico library of

$13213.8 \times 10^{6}$ commercially available small molecules was screened to fit the pharmacophore

133 hypothesis using PHASE (Figure 1D) (Dixon et al., 2006). The top 1000 hits of the in silico

134 screen were clustered for diversity and analyzed based on the fit to residues of the HR1 domain,

135 their interactions with residues of the HR2 domain, and their molecular properties. Moreover, a 
136 number of filters (e.g. elimination of hits with poor ADMET properties using Qikprop) were

137 applied to provide hits with drug-like properties (Duffy et al., 2000). Finally, a subset of 18

138 compounds was selected for experimental validation based on their fit to the pharmacophore

139 hypothesis and molecular diversity of their scaffolds (Figures 1D, 1E).

To evaluate the capacity of the selected hits to promote a conformational activation of

141 Mfn2, a cell-based screening assay was developed based on a FRET biosensor of Mfn2, a

142 molecular tool that we had previously used to monitor the conformational changes of Mfn2 in

143 cells (Franco et al., 2016). Based on the structural model of human Mfn2 in the anti-tethering

144 conformation and in agreement with the crystal structure of Mfn1, the N-terminus is in

145 approximate distance with the C-terminus in the HR2 domain. Therefore the N-terminus was

146 fused with mCerulean and the C-terminus in the HR2 domain was fused with mVenus (Figure

147 2A). This FRET-based Mfn2 biosensor was transiently transfected in HEK 293T cells and co-

148 localized with ATP synthase subunit beta (ATP5B), consistent with mitochondrial localization

149 (Figure S1B). Loss of intra-molecular HR1-HR2 interactions leads to a conformational change

150 and promotes the pro-tethering conformation of $\mathrm{Mfn} 2$. The increased distance between the N-

151 terminus and the C-terminus, occurring in the pro-tethering conformation of Mfn2, is expected to

152 lead to a decrease in the resonance energy that is transferred (Figure 2A). Indeed, we previously

153 showed that the HR1-derived peptide: 367-384 Gly that promotes the pro-tethering conformation

154 of Mfn2 was able to decrease FRET, whereas the HR1-derived peptide: 398-418 Gly that

155 promotes the anti-tethering conformation of Mfn2 was able to increase FRET (Franco et al.,

156 2016). Therefore, we used the HR1-derived peptide: 367-384 Gly as a positive control in our

157 assay. Next, we examined the ability of the 18 putative MASMs to promote the pro-tethering

158 conformation of Mfn2, using a decrease in FRET as a readout (Figure 2B, Table S1). Strikingly, 
159 MASM7 was the only small molecule in our screen that significantly decreased FRET at $10 \mu \mathrm{M}$,

160 indicative of promoting the pro-tethering conformation of Mfn2 (Figure 2B). MASM7 was

161 resynthesized to document its purity by ${ }^{1} \mathrm{H}$ and ${ }^{13} \mathrm{C}$ NMR and reconfirm its activity (Figures $2 \mathrm{C}$

162 and $\mathrm{S} 2 \mathrm{~A})$.

163 MASM7 possesses functional groups that fulfill the 5 criteria of pharmacophore

164 hypothesis (Figure 2D). Specifically, the substituted thiophene ring mimics the side chain of

165 His380 and the hydrophobic methyl and the cyclopropane groups mimic the side chains of

166 Met376 and Val372, respectively. We evaluated the importance of each functional group using

167 the FRET-based Mfn2 biosensor (Figure S2B). Loss of the thiophene ring diminished

168 significantly the ability of this scaffold to reduce FRET. Moreover, the replacement of the

169 methyl group of MASM7 with hydrogen decreased the potency of the compound. Replacement

170 of the cyclopropane group of MASM7 with bulky substituents (e.g. benzene, methyl-furan) was

171 not tolerated, while the replacement with the furan group was well tolerated. These data

172 suggested that the aforementioned functional groups of MASM7 play a key role in its

173 interactions with HR2 to promote a conformational change on Mfn2.

174 Previous structural studies reported that the HR2 domain adopts a helical conformation in

175 the anti-tethering and pro-tethering conformations of Mfn2 (Koshiba et al., 2004; Franco et al.,

176 2016; Cao et al., 2017). MASM7 was designed to bind the HR2 domain and may bind to the

177 proposed residues on HR2 domain in either anti- or pro-tethering conformation (Figures 1A and

178 1B). Therefore, to validate that MASM7 directly interacts with the HR2 domain in vitro, we

179 produced for the first time ${ }^{15} \mathrm{~N}$-labeled Mfn2-HR2 domain (residues 678-757) and recorded

180 heteronuclear quantum spin correlation (HSQC) spectra with or without MASM7 (Figures 2E

181 and S2C). HSQC NMR spectra of the isolated HR2 domain showed evidence of a folded 
182 conformation within HR2. Titration of MASM7 revealed peak broadening corresponding to a

183 number of HR2 residues, demonstrating direct binding to the HR2 domain indicative of a low $184 \mu \mathrm{M}$ binding affinity (Figures $2 \mathrm{E}$ and S2C). On the other hand, titration of MASM7 to an ${ }^{15} \mathrm{~N}-$ 185 labeled Mfn2-HR2 D725A/L727A, mutations that are predicted to disrupt MASM7 binding 186 (Figure 1B), in fact demonstrated no evidence of MASM7 binding even at $200 \mu \mathrm{M}$ (Figures 2F 187 and S2D). Thus, these NMR data suggest that MASM7 binds directly to the HR2 domain of 188 Mfn2 and that D725 and L727 residues are critical for this interaction, consistent with our 189 pharmacophore model.

To further validate that MASM7 interacts with the HR2 in cells, we also generated the 191 same double mutant (D725A/L727A) in the FRET-based Mfn2 biosensor. Wild type (WT) and 192 D725A/L727A FRET-based Mfn2 biosensors presented similar acceptor/donor ratios, suggesting 193 that these double point mutations did not alter structural transformation of Mfn2 between pro194 and anti-tethering conformations (Figure 2G). Titration of MASM7 reduced FRET in a 195 concentration-dependent manner. Of note, the D725A/L727A Mfn2 biosensor required almost 196 twice the concentration of MASM7 $\left(\mathrm{IC}_{50}=55 \mu \mathrm{M}\right)$ for the reduction of FRET compared to the 197 WT biosensor $\left(\mathrm{IC}_{50}=31 \mu \mathrm{M}\right)$ (Figure $\left.2 \mathrm{H}\right)$. Therefore, our results further validated the 198 interaction of MASM7 with D725 and L727 on the HR2 domain of Mfn2 and the promotion of 199 pro-tethering conformation by MASM7.

200 Next, we examined if the pro-tethering activity of MASM7 can also trigger mitochondrial 201 fusion in cells. We examined whether MASM7 could induce mitochondrial fusion in wild type 202 MEFs and MEFs lacking Mfn1, Mfn2, or both, using mitochondrial aspect ratio (mitochondrial 203 length/width, reflecting fusion) as a readout. Notably, MASM7 increased significantly 204 mitochondrial aspect ratio in WT MEFs. The same was observed, albeit to a lesser degree, in 
MFN1 KO and MFN2 KO MEFs (Figures 3A, B and C). In contrast, MASM7 did not increase mitochondrial aspect ratio in MFN1/MFN2 double knockout (DKO) MEFs. Collectively, these data indicate that MASM7 can induce mitochondrial fusion by directly activating either Mfn2 or Mfn1 and MASM7-induction of mitochondrial fusion is greater when both Mfn2 and Mfn1 are expressed. This result is in agreement with the high sequence homology between Mfn1 and Mfn2 and our pharmacophore model hypothesis for Mfn2 activation based on conserved residues

211 that regulate HR1-HR2 interactions (Figure S1). Furthermore, it is consistent with the work by

212 Nunnari and colleagues that suggests heterotypic complexes possess greater efficiency in fusion

213 compared to homotypic Mfn1 or Mfn2 complexes (Hoppins et al., 2011) Strikingly, MASM7

214 emerged as a potent inducer of fusion, achieving an $\mathrm{EC}_{50}$ approximately four times lower than

215 367-384 Gly (MASM7 EC $50=75$ nM; 367-384 Gly EC $_{50}=340$ nM) (Figure 3D). Furthermore,

216 while MASM7 markedly increased mitochondrial aspect ratio in MFN2 KO MEFs transduced

217 with WT Mfn2, MASM7 did not increase mitochondrial aspect ratio in MFN2 KO MEFs

218 transduced with D725A/L727A Mfn2, underscoring the specificity of MASM7 (Figures 3E and

$2193 \mathrm{~F})$. Taken together, the specificity of the MASM7-mitofusin interaction is evidenced by

220 mutagenesis using NMR, FRET and mitochondrial fusion studies (Figures 2E, 2F, 2H and 3F).

\section{Conclusions}

223 In conclusion, we have identified the first direct small molecule activator of mitofusins using a

224 pharmacophore model small-molecule screen based on the interaction of HR1-HR2 domains of

225 Mfn2. Our studies support the concept that disruption of HR1-HR2 interactions is critical for 226 mitochondrial tethering and fusion to take place. MASM7 promptly and robustly promotes 227 mitochondrial fusion in a mitofusin-dependent manner. The ability to directly activate mitofusins 
228 with small molecules provides new opportunities to elucidate the precise roles of these proteins

229 in mitochondrial dynamics as well as the role of mitochondrial fusion in fundamental

230 physiological processes. As importantly, the discovery of MASM7 raises the possibility of novel

231 therapeutics. These may be applied to orphan syndromes such as Charcot-Marie-Tooth disease

232 type 2 a resulting from mutation in one $\mathrm{Mfn} 2$ allele, as we have recently shown (Rocha et al.,

233 2018). Moreover, direct mitofusin activation may also present therapeutic opportunities for

234 common acquired syndromes such as heart failure, neurodegeneration, cancer, and diabetes in

235 which functional defects in mitochondrial fusion contribute to pathogenesis (Shirihai et al., 2015;

236 Schrepfer et al., 2016).

\section{Acknowledgements}

239 We thank Louis Hodgson for his help with FRET studies and David Chan for providing MEF

240 cell lines. Studies were supported by NIH grants 1R01CA178394 (E.G.) and 5R01HL128071

241 (R.N.K.). Support was also provided by NIH awards 1S10OD016305, P30CA013330 and

242 American Heart Association Collaborative Science Award (E.G. and R.N.K).

\section{Competing Financial Interests}

245 The authors declare no competing financial interests. 


\section{References}

248

249

250

251

252

253

254

255

256

257

258

259

260

261

262

263

264

265

266

267

268

269

1. Baell, J., Walters, M.A. (2014). Chemistry: Chemical con artists foil drug discovery. Nature 513, 481-483.

2. Bombelli, F. et al. (2014). Charcot-Marie-Tooth disease Type 2A From Typical To Rare Phenotypic and Genotypic Features. JAMA Neurol. 71, 1036-1042.

3. Cao, Y.L. et al. (2017). MFN1 structures reveal nucleotide-triggered dimerization critical for mitochondrial fusion. Nature 542, 372-376.

4. Chen, H., Chomyn A., Chan, D.C. (2005). Disruption of fusion results in mitochondrial heterogeneity and dysfunction. J. Biol. Chem. 280, 26185-26192.

5. Delaglio, F., Grzesiek, S., Vuister, G.W., Zhu, G., Pfeifer, J., Bax, A. (1995). NMRPipe: A multidimensional spectral processing system based on UNIX pipes. J. Biomol. NMR 6, 277-293.

6. Detmer, S.A., Chan, D.C. (2007). Functions and dysfunctions of mitochondrial dynamics. Nat. Rev. Mol. Cell Biol. 8, 870-879.

7. Dixon, S.L., Smodyrev, A.M., Knoll, E.H., Shashidhar, N.R., Shaw, D.E., Freisner R. A. (2006). Phase: a new engine for pharmacophore perception, 3D QSAR, model development, and 3D database screening: 1. Methodology and preliminary results. J. Comput. Aided Mol. Des. 20, 647-671.

8. Duffy, E. M., Jorgensen, W.L. (2000). Prediction of properties from simulations: free energies of solvation in hexadecane, octanol and water. J. Am. Chem. Soc. 122, 28782888.

9. Franco, A. et al. (2016). Correcting mitochondrial fusion by manipulating mitofusin conformations. Nature 540, 74-79. 
10. Hoppins, S., Edlich, F., Cleland, M. M., Banerjee, S., McCaffery, J. M., Youle, R. J., Nunnari, J. (2011). The soluble form of BAX regulates mitochondrial fusion via MFN2 homotypic complexes. Mol. Cell 41, 150-160.

11. Huang, P., Galloway, C. A., Yoon, Y. (2011). Control of mitochondrial morphology through differential interactions of mitochondrial fusion and fission proteins. PLoS ONE $6, \mathrm{e} 20655$.

12. Koshiba, T., Detmer, S.A., Kaiser, J.T., Chen, H., McCaffery, J.M., Chan, D.C. (2004). Structural basis of mitochondrial tethering by mitofusin complexes. Science 305,858 862.

13. Mattie, S., Riemer, J., Wideman, J.G., McBride, H.M. (2018). A new mitofusin topology places the redox-regulated $\mathrm{C}$ terminus in the mitochondrial intermembrane space. J. Cell Biol. 217, 507-515.

14. Nagai, T., Ibata, K., Park, E.S., Kubota, M., Mikosiba, K., Miyawaki, A. (2002). A variant of yellow fluorescent protein with fast and efficient maturation for cell-biological applications. Nat. Biotechnol. 20,87-90.

15. Rizzo, M. A., Springer G.H., Granda, B., Piston, D.W. (2004). An improved cyan fluorescent protein variant useful for FRET. Nat. Biotechnol. 22, 445-449.

16. Rocha, A. G. et al. (2018) Mfn2 agonists reverse mitochondrial defects in preclinical models of Charcot Marie Tooth disease type 2A. Science, in press.

17. Sastry, M., Lowrie, J.F., Dixon S.L., Sherman, W. (2010). Large-scale systematic analysis of $2 \mathrm{~d}$ fingerprint methods and parameters to improve virtual screening enrichments. J. Chem. Inf. Model. 50, 771-784. 
18. Schrepfer, E., Scorrano, L. (2016). Mitofusins, from mitochondria to metabolism. Mol. Cell 61, 683-694.

19. Shirihai, O. S., Song, M., Dorn, G.W. (2015). How mitochondrial dynamism orchestrates autophagy. Circ. Res. 116, 1835-1849.

20. Vranken, W. F. et al. (2005). The CCPN data model for NMR spectroscopy: Development of a software pipeline. Proteins 59, 687-696. protein structure and function prediction. Nat. Methods 12, 7-8. mitochondria. Science 34, 471-475. 


\section{Methods}

\section{Structural Model of Mfn2}

304 The structural model of Mfn2 was calculated based on the I-TASSER (Iterative Threading 305 ASSEmbly Refinement) hierarchical approach to protein structure as we previously described

306 (Yang et al., 2015; Franco et al., 2016). Top solutions derived from I-TASSER approach were 307 based on the bacterial dynamin-like protein structure in the PDB (ID: 2J69). Energy 308 minimization and analysis of the top-ranked structure was performed with MAESTRO tools 309 (Maestro, v10.5, Schrödinger, LLC). PyMOL (The PyMOL Molecular Graphics System. Version 310 1.8; Schrödinger, LLC) was used for preparing the figures.

\section{$311 \quad$ Ligand preparation library}

312 eMolecules (www.emolecules.com) library of purchasable compounds was converted to 3D 313 structures using LIGPREP (LigPrep, version 3.8, Schrödinger, LLC) and EPIK (Epik, version 314 3.6, Schrödinger, LLC) generating an in silico library of approximately 13.8 million compounds 315 containing compounds with different ionization state at $\mathrm{pH} 7.0 \pm 2.0$, stereochemistry and 316 tautomeric form, excluding potential Pan Assay Interference Compounds (PAINS) using PAINS

317 definitions included in Canvas (Baell et al., 2014). Conformation analysis of ligands was 318 calculated using the OPLS3 force field.

3D Pharmacophore model generation and screen

321 Phase (Phase, version 4.6, Schrödinger, LLC) module was used to generate a pharmacophore 322 hypothesis and a 3D pharmacophore screen (Dixon et al., 2006). The coordinates of the HR1 323 helix residues Val372, Met376 and His380 from the structural model of Mfn2 were used to 324 assign pharmacophore points in 3D coordinates. Pharmacophore hypothesis included 5 features 
325 as defined in Phase for 3 hydrophobic groups to mimic the sidechain residues of Val273 and

326 Met376 and an aromatic ring with a hydrogen-bond donor to mimic the sidechain of His380. The

327 pharmacophore screen used the in silico library of compounds prepared from the eMolecules

328 library in pre-existing conformations with the requirement to satisfy at least 4 out of the 5

329 pharmacophore features of the hypothesis. The top 1000 compounds ranked based on the Phase

330 Score were selected for further visual analysis and clustered for diversity using dendritic

331 fingerprints in Canvas (Sastry et al., 2010). Physicochemical and AMDET properties including

332 Lipinski rules, permeability, $\log \mathrm{P}$, metabolic liabilities and hERG inhibition were evaluated

333 using QikProp (QikProp, version 3.4, Schrödinger, LLC, New York, NY, 2011). The highest 8

334 ranked compounds and the 10 most diverse compounds yielded selected molecules for

335 experimental validation. MASM7 was checked for potential Pan Assay Interference Compounds

336 (PAINS) (Baell et al., 2014) and has not been reported as a hit in previous screens in Pubchem

337 database.

339 Compounds

340 Pro-fusion 367-384 Gly peptide, QIAEVRGIMDSLHMAARGGYGRKKRRQRRR was

341 synthesized, purified at $>95 \%$ purity by Life Technologies. MASM7 compound was obtained

342 from Enamine (cat. \# EN300-396282). Screened MASMs were purchased from Enamine,

343 ChemBridge and ChemDiv. All compounds were $>95 \%$ pure, dissolved in DMSO to prepare a

$34410 \mathrm{mM}$ stock solution.

$346 \quad$ Cell lines 
Cell lines were purchased from ATCC. MEFs (WT, Mfn1 KO, Mfn2 KO and Mfn1/Mfn2 DKO) were also provided by David Chan's laboratory. HEK 293T cells were provided by Louis Hodgson's laboratory. All cells maintained in DMEM (Life Technologies) supplemented with $10 \% \mathrm{FBS}, 100 \mathrm{U} \mathrm{ml}^{-1}$ penicillin/streptomycin and $2 \mathrm{mM} \mathrm{L-glutamine.}$

Molecular cloning

To generate FRET-based Mfn2 biosensor construct, we utilized pcDNA3.1-Flag-Mfn2 (5'Flag epitope-tagged human Mfn2) as backbone, and fused mCerulean (cyan fluorescent protein; CFP) and mVenus (yellow fluorescent protein; YFP) onto the 5' and 3' ends respectively of the Mfn2 open reading frame (Nagai et al., 2002; Rizzo et al., 2004; Yun et al., 2013). The construct was confirmed by sequencing. FRET-based Mfn2 biosensor mutant D725A/L727A was generated by site-directed mutagenesis of the wild type probe.

\section{FRET assay}

361 HEK 293 T cells $\left(5 \times 10^{5}\right.$ cells/well $)$ were seeded in a pre-coated with poly L-lysine 6-well clear

362 bottom plate. Cells were transfected with the total amount of $2.5 \mu \mathrm{g}$ of DNA per well for the 363 indicated Mfn2 FRET constructs using Lipofectamine ${ }^{\circledR} 3000$ Reagent (Invitrogen) according to 364 the manufacturer's protocol. The following day, transfected cells were transferred to a pre-coated

365 with poly L-lysine 96-well black plate $\left(2 \times 10^{4}\right.$ cells/well). The following day, cell media was 366 replaced with FluoroBrite ${ }^{\mathrm{TM}}$ DMEM (Invitrogen) supplememented with $10 \% \mathrm{FBS}, 100 \mathrm{U} \mathrm{ml}^{-1}$ 367 penicillin/streptomycin, $2 \mathrm{mM}$ L-glutamine and $1 \mathrm{mM}$ GTP $\gamma \mathrm{S} 15 \mathrm{~min}$ prior treatments with 368 MASMs. GTP $\gamma$ S (Sigma Aldrich) was used to inhibit GTPase activity of Mfn2. Subsequently, 369 cells were treated for $2 \mathrm{~h}$ with MASMs or vehicle (0.1\% DMSO). Dilutions of MASMs were 370 performed using a TECAN D300e Digital Dispenser from $10 \mathrm{mM}$ stock solutions. Fluorescence 
371 intensity was detected by a M100 microplate reader (TECAN), filters that were used for the

372 FRET studies: donor (mCerulean) Ex: 436 nm/Em: 480 nm, acceptor (mVenus) Ex: 436 nm/Em:

$373535 \mathrm{~nm}$. Acceptor/Donor ratio referred to the text and figures correspond to the ratio of

374 fluorescence intensities (relative fluorescence units: RFU) detected by the acceptor filter/donor

375 filter. Non-transfected cells were used to subtract background fluorescence from each of the

376 aforementioned filters that were used in the study.

$378 \quad$ NMR samples and experiments

379 Mfn2 residues 678-757 corresponding to the HR2 domain were cloned into a pET-28 vector and 380 transformed into BL21(DE3) CodonPlus (DE3)-RIPL E. coli cells. The double mutant 381 D725A/L727A construct was also prepared by site-directed-mutagenesis. Cells were grown at $38237^{\circ} \mathrm{C}$ in $1 \mathrm{~L}$ of $\mathrm{LB}$ media to an $\mathrm{OD}_{600}$ of 0.8 , cells were then harvested and resuspended in $1 \mathrm{~L}$ of 383 M9 media supplemented with $1.5 \mathrm{gr} / \mathrm{L}$ of ${ }^{15} \mathrm{~N}$ ammonium chloride grown for 45 min at $37^{\circ} \mathrm{C}$ and 384 induced at $18^{\circ} \mathrm{C}$ for 16 hours with $1 \mathrm{mM}$ IPTG. MFN2 HR2 domain was purified from bacterial 385 pellets by high-pressure homogenization in lysis buffer $(20 \mathrm{mM}$ Tris. $\mathrm{HCl} \mathrm{pH}$ 7, $250 \mathrm{mM} \mathrm{KCl,} 25$ $386 \mathrm{mM}$ imidazole, and Roche complete EDTA free protease inhibitor cocktail) and ultracentrifuged 387 at 45,000 $\mathrm{g}$ for $45 \mathrm{~min}$. The supernatant was applied to pre-equilibrated $1 \mathrm{~mL}$ HisPur Ni-NTA 388 Resin washed in lysis buffer and eluted using elution buffer (20 mM Tris.HCl pH 6, $250 \mathrm{mM}$ $389 \mathrm{KCl}, 400 \mathrm{mM}$ imidazole). ${ }^{15} \mathrm{~N}-\mathrm{MFN} 2-\mathrm{HR} 2$ was further purified by size exclusion 390 chromatography (Superdex 75 Increase 10/300 GL column) in gel filtration buffer (20 mM 391 potassium phosphate $\mathrm{pH} 6,150 \mathrm{mM} \mathrm{KCl}$ ). Fractions containing the ${ }^{15} \mathrm{~N}-\mathrm{MFN} 2-\mathrm{HR} 2$ were 392 confirmed by SDS-PAGE, pooled and concentrated to $50 \mu \mathrm{M}$ in NMR buffer $(20 \mathrm{mM}$ potassium 393 phosphate $\mathrm{pH} 6,150 \mathrm{mM} \mathrm{KCl}, 10 \% \mathrm{D}_{2} \mathrm{O}$ ) using a $10 \mathrm{KDa}$ cut-off Centricon spin concentrator 
394 for NMR analysis. ${ }^{1} \mathrm{H}^{15}{ }^{15}$-HSQC spectra of $50 \mu \mathrm{M}$ MFN2-HR2 in the presence and absence of

395 MASM7 were recorded on a BRUKER AVANCE IIIHD 600MHz system equipped with a 5mm

396 H/F-TCI CryoProbe at $25^{\circ} \mathrm{C}$. Spectra were processed using qMDD (mddnmr v2.0) and

397 NMRPIPE and analyzed using Analysis (CCPNMR) (Vranken et al., 2005; Delaglio et al., 398 1995).

$400 \quad$ Live cell imaging

401 MEFs were seeded on chamber slides (MatTek Corporation: $35 \mathrm{~mm}$ dishes, No. 1.5, $14 \mathrm{~mm}$ 402 glass diameter) at $\sim 70 \%$ confluence. Cells were treated with MASM7 or 367-384 Gly (1 $\mu$ M, 2 403 h). Cells were stained with MitoTracker Green (200 nM, Invitrogen) for $20 \mathrm{~min}$ at $37^{\circ} \mathrm{C}$. After 404 treatments, media was replaced with FluoroBrite ${ }^{\mathrm{TM}}$ DMEM (Invitrogen) supplemented with 10\% 405 FBS, $100 \mathrm{U} \mathrm{ml}^{-1}$ penicillin/streptomycin, $2 \mathrm{mM} \mathrm{L-glutamine}$ and MASM7 or 367-384 Gly (1 $406 \mu \mathrm{M}$ ) prior image acquisition. Images were taken with Leica SP5 inverted confocal microscope.

407 Data were analyzed with Image J software. $>200$ mitochondria were measured per condition in 408 mitochondrial aspect ratio.

$410 \quad$ Immunofluorescence

411 Transfected HEK 293 T cells were washed with once PBS and fixed with 2\% PFA for 13 min.

412 Subsequently, cells were blocked, then incubated for $1 \mathrm{~h}$ at room temperature with the primary 413 antibody for ATP5B (Abcam; ab128743). After incubation with the primary antibody, cells were 414 washed with PBS and incubated for $1 \mathrm{~h}$ at room temperature with the secondary antibody 415 (ThermoFisher Scientific; A11010). Images were taken with Leica SP5 inverted confocal 416 microscope or Zeiss fluorescent microscope. Data were analyzed with Image J software. 
bioRxiv preprint doi: https://doi.org/10.1101/301713; this version posted April 17, 2018. The copyright holder for this preprint (which was not certified by peer review) is the author/funder. All rights reserved. No reuse allowed without permission.

418

\section{Figure Legends}

A

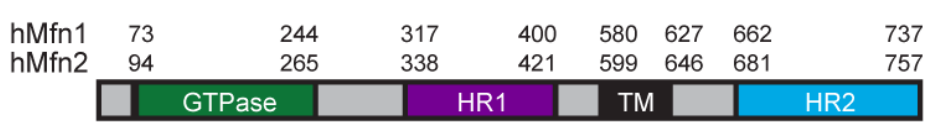

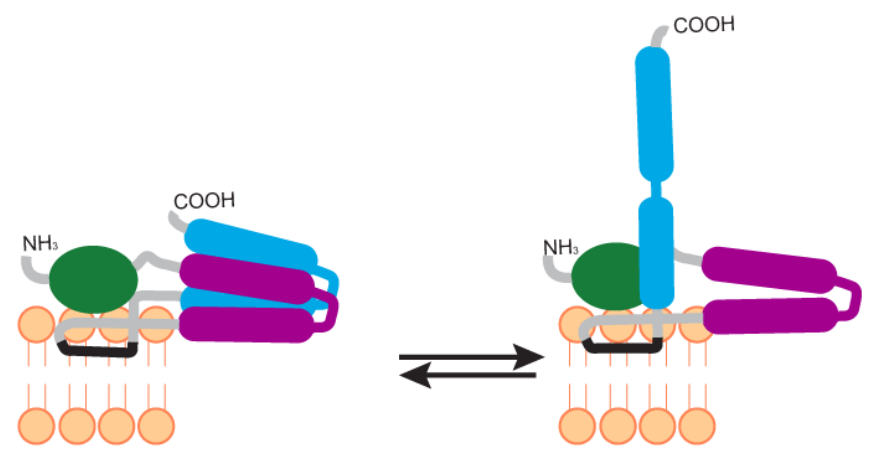

Anti-tethering conformation

Pro-tethering conformation

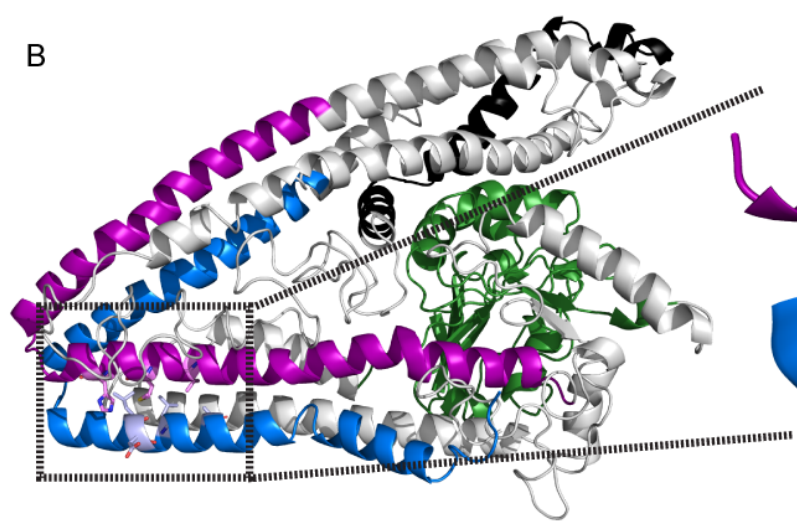

C

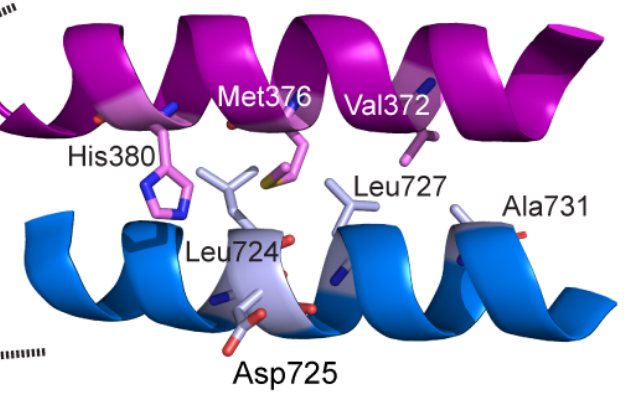

D

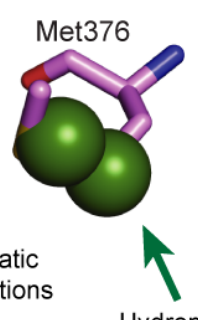

Hydrophobic interactions

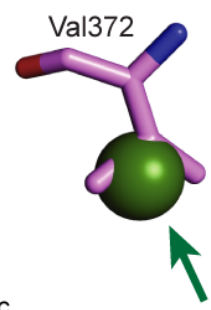

Hydrophobic interactions

$E$

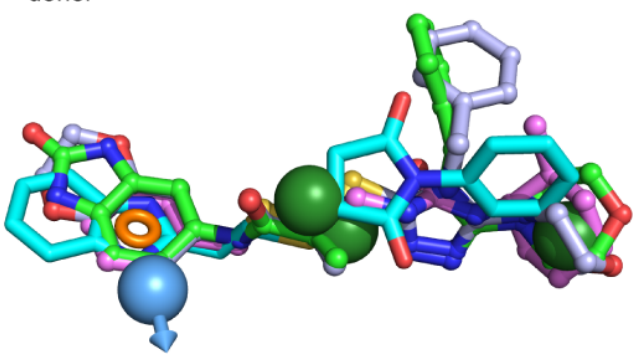


421 Figure 1. Pharmacophore model-based strategy for in silico discovery of MASMs. (A) Mfn2

422 undergoes conformational changes to promote mitochondrial tethering. Anti- and pro-tethering

423 conformations of Mfn2. (B) Ribbon representation of the human Mfn2 structural model and

424 HR1-HR2 interactions. (C) Pharmacophore hypothesis based on the interactions of the side

425 chains of the HR1-amino acids: Val372, Met376, His380. (D) Schematic representation of a in

426 silico-based screening strategy for the discovery of putative MASMs. (E) A set of small

427 molecules shown in different color demonstrates how selected molecules fulfill the criteria of the

428 pharmacophore hypothesis.

429 
A
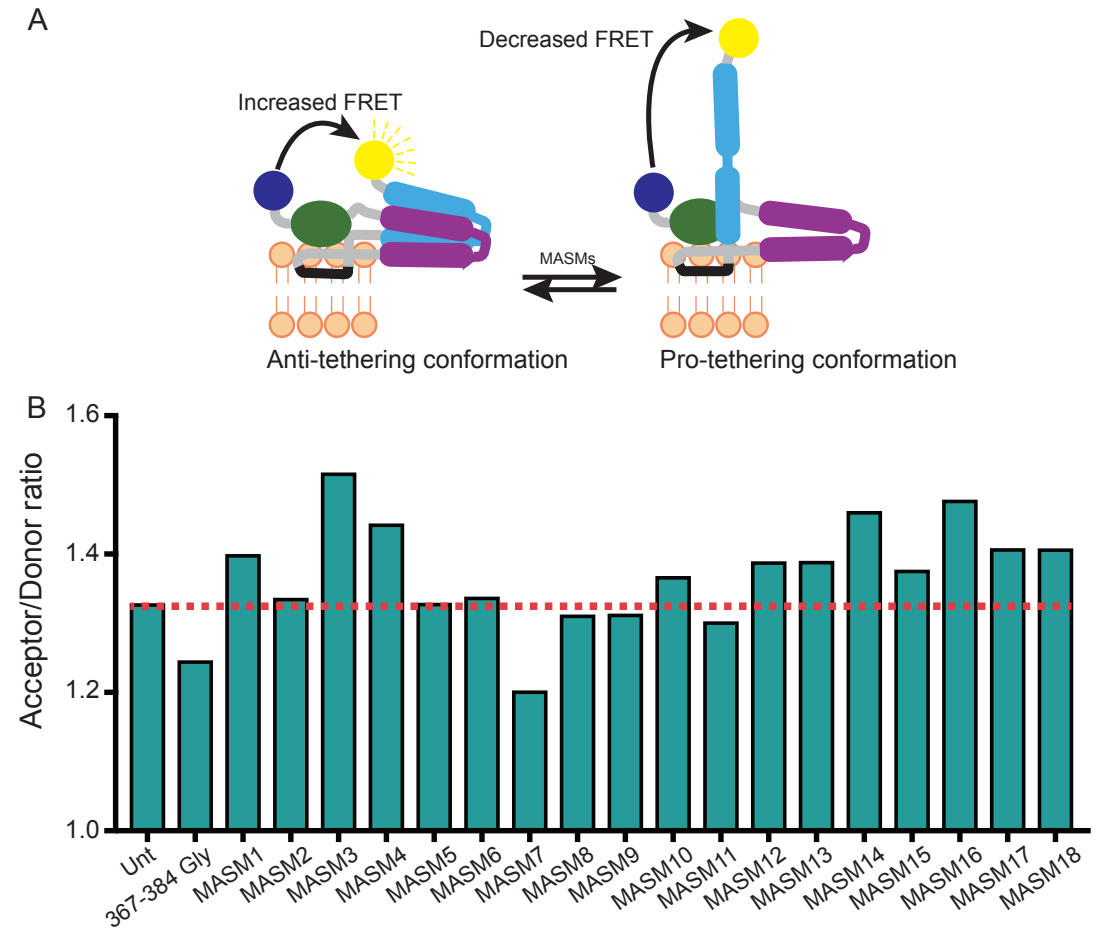

C
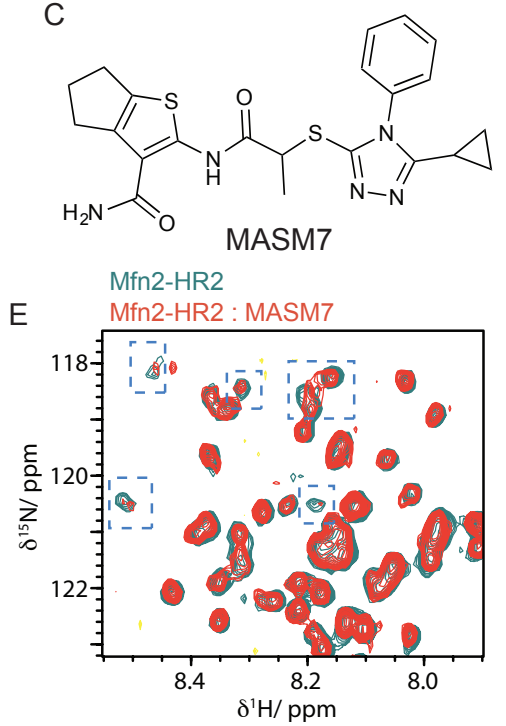

G

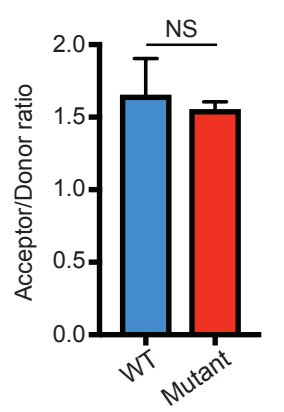

D
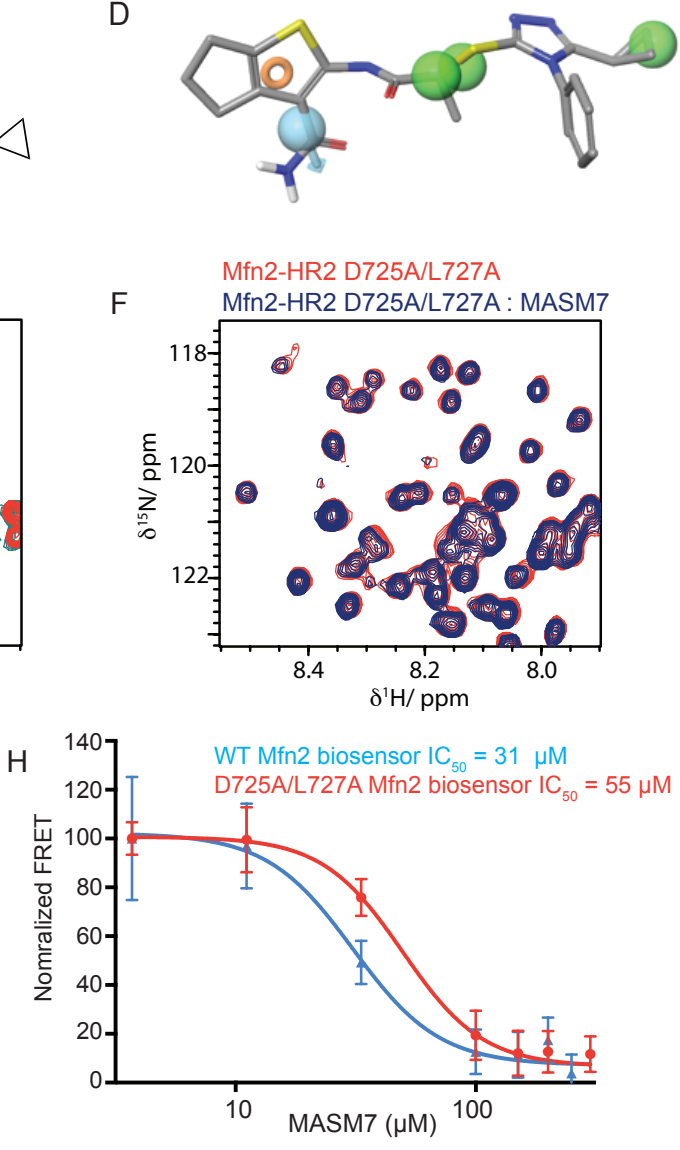
Figure 2. MASM7 promotes the pro-tethering conformation of Mfn2 through binding to

433 the HR2 domain. (A) Schematic representation of FRET-based Mfn2 biosensor conformational

434 changes. Cyan sphere represents mCerulean and yellow sphere represent mVenus. (B) Screening

435 of putative MASMs using FRET-based Mfn2 biosensor in HEK 293T cells. Cells were treated

436 with MASMs or 367-384 Gly (10 $\mu \mathrm{M}, 2 \mathrm{~h})$. We used 367-384 Gly peptide as a positive control

437 (Franco et al., 2016). (C) Structure of MASM7. (D) MASM7 satisfies the pharmacophore

438 hypothesis features as described in our methodology. Green spheres represent hydrophobic

439 interactions of the cyclopropane and methyl groups. Cyan sphere represents hydrogen bond

440 interaction of the amide group and the orange ring represents aromatic interaction of the

441 thiophene group. (E) Zoomed region of overlaid ${ }^{1} \mathrm{H}^{15} \mathrm{~N}$ HSQC spectra of Mfn2-HR2 $(50 \mu \mathrm{M})$

442 with (red cross-peaks) and without (green cross-peaks) MASM7 (200 $\mu \mathrm{M})$. Line broadening and

443 chemical shifts upon addition of MASM7 are highlighted with blue boxes. (F) Zoomed region of

444 overlaid ${ }^{1} \mathrm{H}_{-}{ }^{15} \mathrm{~N}$ HSQC spectra of Mfn2-HR2 D725A/L727A mutant $(50 \mu \mathrm{M})$ with (blue cross-

445 peaks) and without (red cross-peaks) MASM7 $(200 \mu \mathrm{M})$. Effects of MASM7 on broadening and

446 chemical shifts are lost. See full spectra in (Figure S5). (G) Acceptor/Donor ratios of WT and

447 D725A/L727A FRET based Mfn2 biosensors. Error bars represent mean $\pm \mathrm{SD}, n=3$. (H)

448 D725A/L727A FRET based Mfn2 biosensor decreased potency of MASM7. Error bars represent

449 mean \pm SEM, $n=4$. 


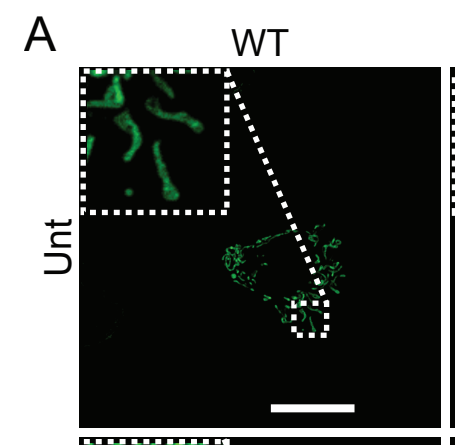

MFN1 KO
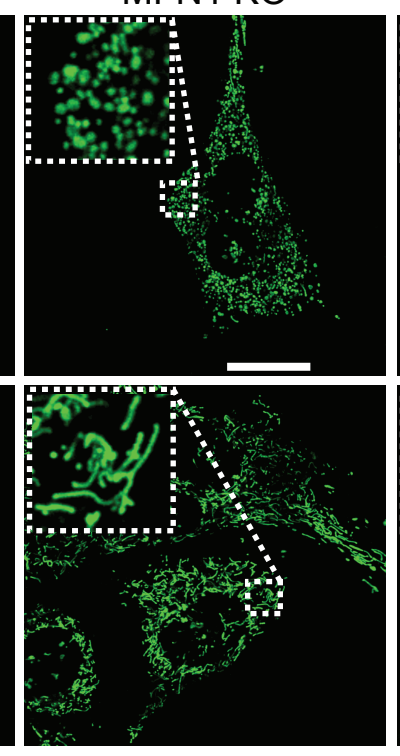

C
MFN2 KO
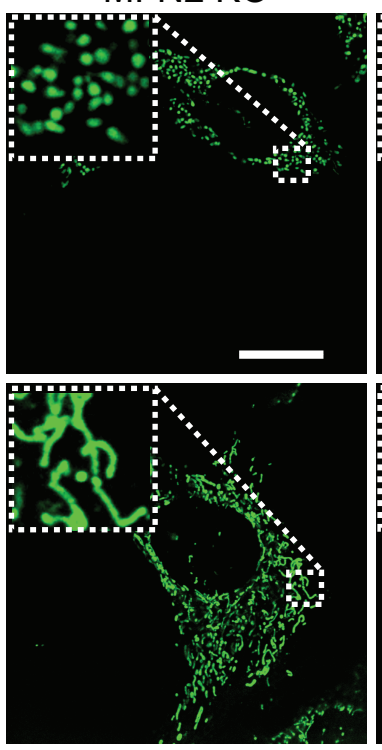

B

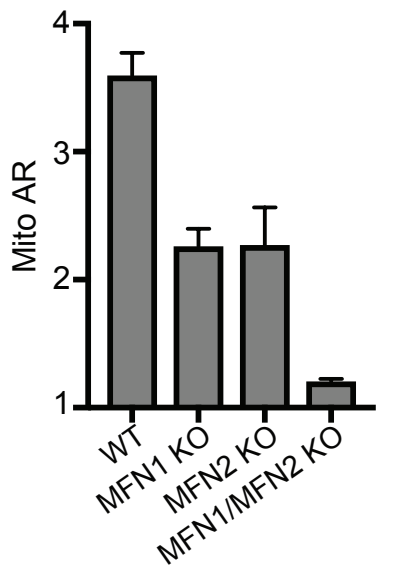

D

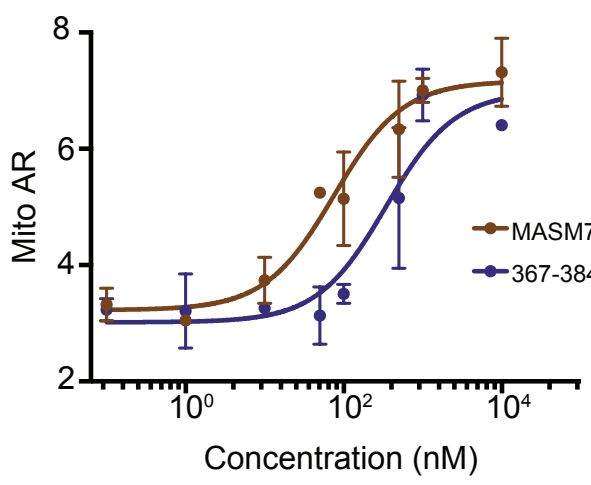

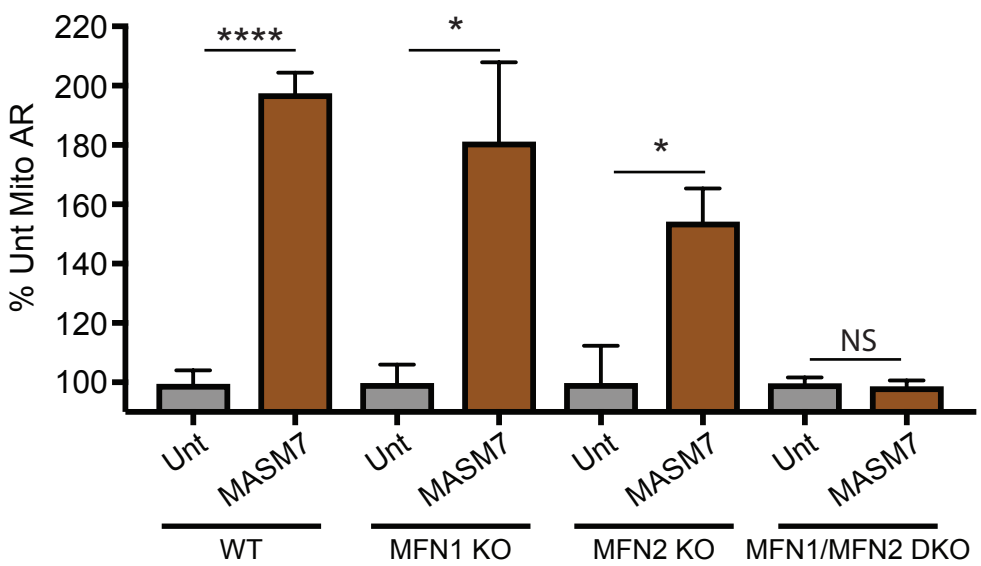

E

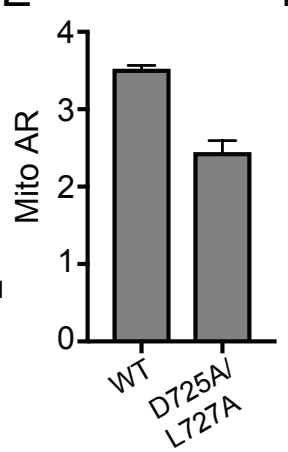

MFN1/MFN2 DKO
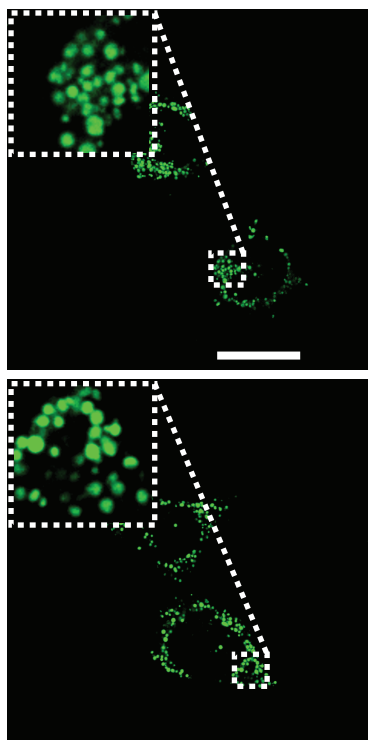

MFN2 KO MFN1/MFN2 DKO

$\mathrm{F}$

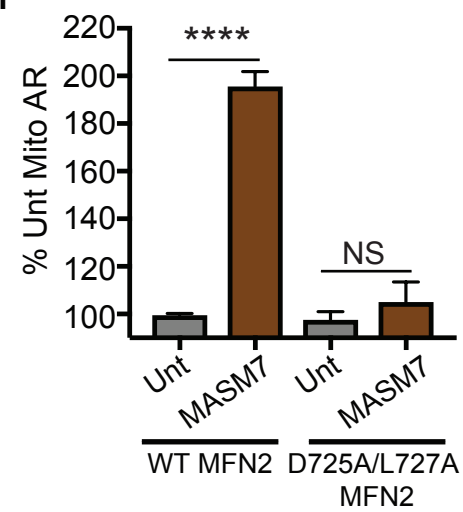


453 MASM7 induced mitochondrial fusion in WT, MFN1 KO and MFN2 KO MEFs, but not in

454 MFN1/MFN2 DKO MEFs. Cells were treated with MASM7 (1 $\mu \mathrm{M}, 2 \mathrm{~h})$. Mitotracker green

455 stains mitochondria. Scale bar $20 \mu \mathrm{m}$. Zoomed images are 5x. (B) Quantification of

456 mitochondrial aspect ratio (Mito AR) in untreated WT, MFN1 KO, MFN2 KO MEFs and

457 MFN1/MFN2 DKO MEFs. Error bars represent mean $\pm \mathrm{SD}, n=3$. $>200$ mitochondria were

458 scored per condition. (C) Relative increase of Mito AR in the indicated cells upon MASM7

459 treatment. Error bars represent mean $\pm \mathrm{SD}, n=3 .>200$ mitochondria were scored per condition.

460 (D) MASM7 and 367-384 Gly concentration-responsively increased Mito AR in WT MEFs.

461 Cells were treated with MASM7 or 367-384 Gly at the indicated concentrations for 2 h. Error

462 bars represent mean $\pm \mathrm{SEM}, n=3$. (E) Quantification of mitochondrial aspect ratio (Mito AR) in

463 untreated MFN2 KO MEFs reconstituted with WT MFN2 or D725A/L727A MFN2. Error bars

464 represent mean $\pm \mathrm{SD}, \mathrm{n}=2$. $>200$ mitochondria were scored per condition. (F) Relative increase

465 of Mito AR in MFN2 KO MEFs reconstituted with WT MFN2 or D725A/L727A MFN2 upon

466 MASM7 treatment. Error bars represent mean $\pm \mathrm{SD}, \mathrm{n}=2 .>200$ mitochondria were scored per 467 condition. 
bioRxiv preprint dol: https://doi.org/10.1101/301713; this version posted April 17, 2018. The copyright holder for this preprint (which was not certified by peer review) is the author/funder. All rights reserved. No reuse allowed without permission.

470

471 Supplementary Table 1: Structures of predicted MASMs.

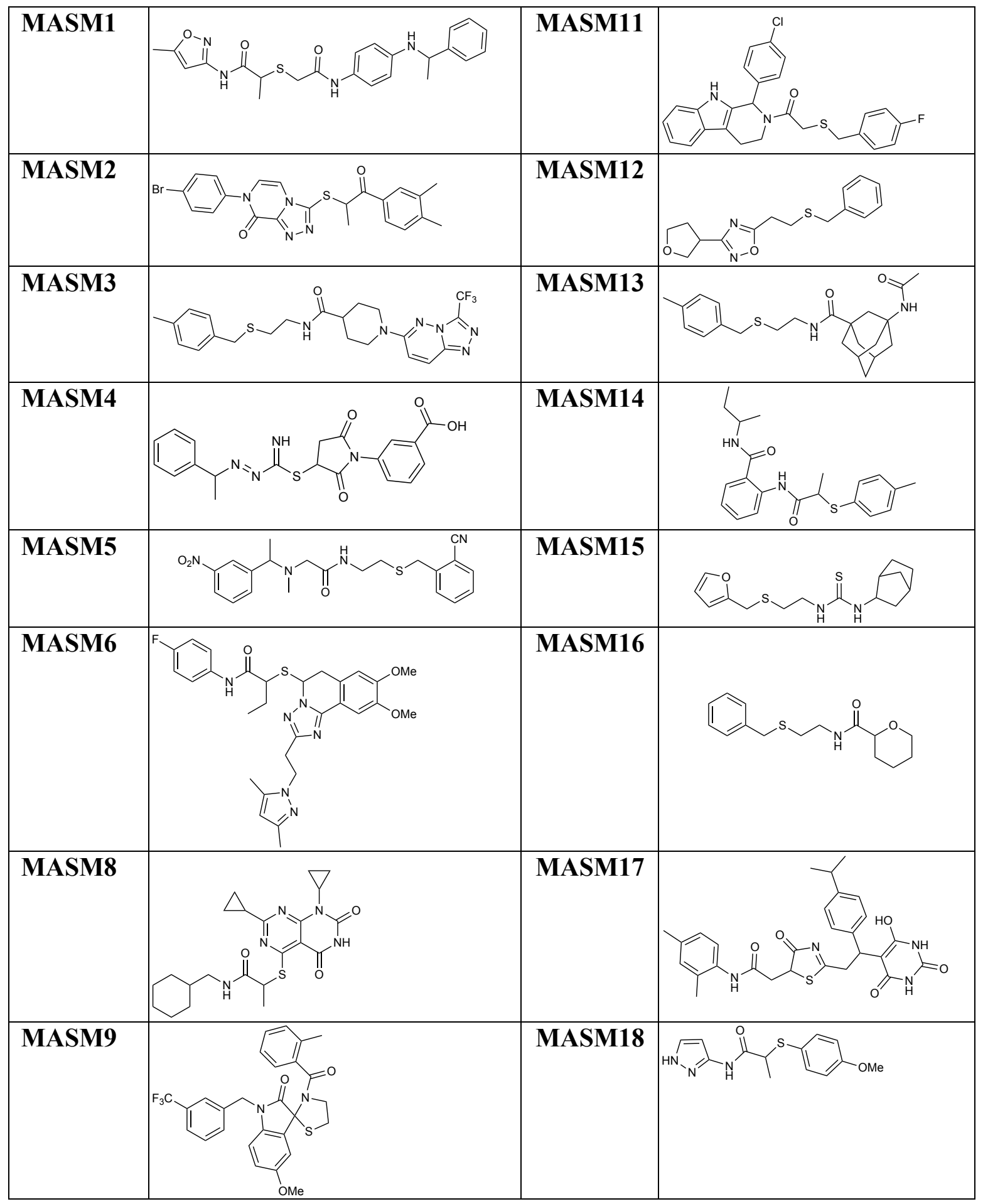

25 
bioRxiv preprint doi: https://doi.org/10.1101/301713; this version posted April 17, 2018. The copyright holder for this preprint (which was not certified by peer review) is the author/funder. All rights reserved. No reuse allowed without permission.

472

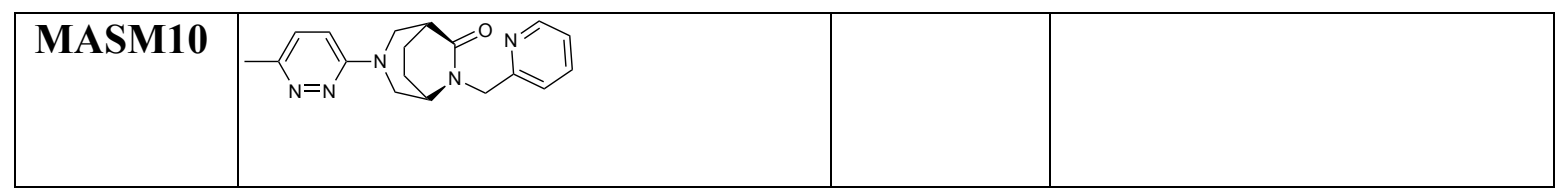

473

474

475

476

477

478

479

480

481

482

483

484

485

486

487

488

489

490

491

492

493 
bioRxiv preprint doi: https://doi.org/10.1101/301713; this version posted April 17, 2018. The copyright holder for this preprint (which was not certified by peer review) is the author/funder. All rights reserved. No reuse allowed without permission.

\section{Supplementary Figures:}

A

\begin{tabular}{ll|l|l|l|l|l|}
\cline { 2 - 4 } Mfn2 & $\square$ & GTPase & & HR1 & TM & \\
\hline
\end{tabular}

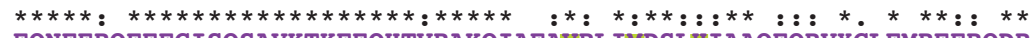
FNFERQFEECISQSAVKTKFEQHTVRAKQIAEAVRLIMDSLHIAAQEQRVYCLEMREERQDR Mfn2_HUMAN FQNFERRFEECISQSAVKTKFEQHTVRAKQIAEAVRLIMDSLHMAAREQQVYCEEMREERQDR Mfn1-MOUSE FQNFEQTFEECISQSAVKTKFEQHTIRAKQILDTVKNILDSVNVAAAEKRVYSMEEREDQIDR Mfn1_RAT FQNFEQTFEECISQSAVKTKFEQHTIRAKQILDTVKNILDSVNVAAAEKRVYSMEEREDQIDR Mfn1-HUMAN FONFEOIFEECISOSAVKTKFEOHTIRAKOILATVKNIMDSVNLAAEDKRHYSVEEREDOIDR

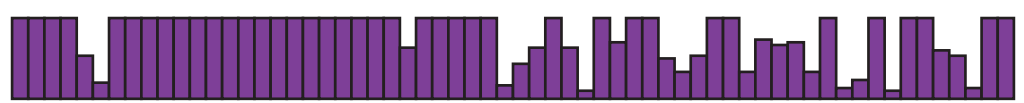

Mfn2 MOUSE Mfn2-RAT Mfn2-HUMAN Mfn1-MOUSE Mfn1-RAT

Mfn1_HUMAN

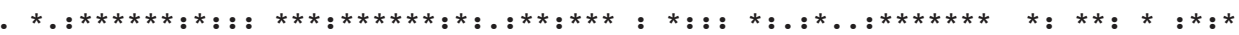
SYTGSNCSHOVOOELSGTFAHLCOOVDITRDNLEOE IAAMNKKVEALDSEOSRAKLLRNKAGWLDSELNMFTHOYLO 77 SYTGSNCSHOVOOELSGTFAHLCOOVDITRDNLEOEIAAMNKKVEA D SOSK KLLRNKAGWLDSELNMF IHOYLO 77 SYTGSNCSHQVQQELSGTFAHLCQQVDVTRENLEQEIAAMNKKIEVLD S QSK KLLRNKAGWLDSELNMFTHQYLQ 77 SFTSANCSHQVQQEMATTFARLCQQVDVTQKHLEEEIARLSKEIDQ KEIONN KLLRNKAVQLESELENFSKQFLH 77 KFTSANCSHOVOOEMATTFARLCOOVDVTOKHLEEEIARLSKEIDO E KONN KLLRNKAVQLERELENFSKOFLH 77 SSTSANCSHQVKQQIATTFARLCQQVDITQKQLEEEIARLPKEIDQ EIQNN KLLRNKAVQLENELENFTKQFLP 77

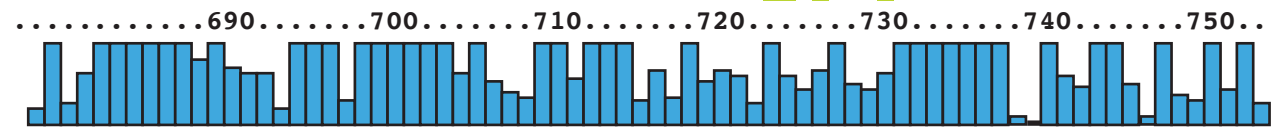

$\mathrm{B}$
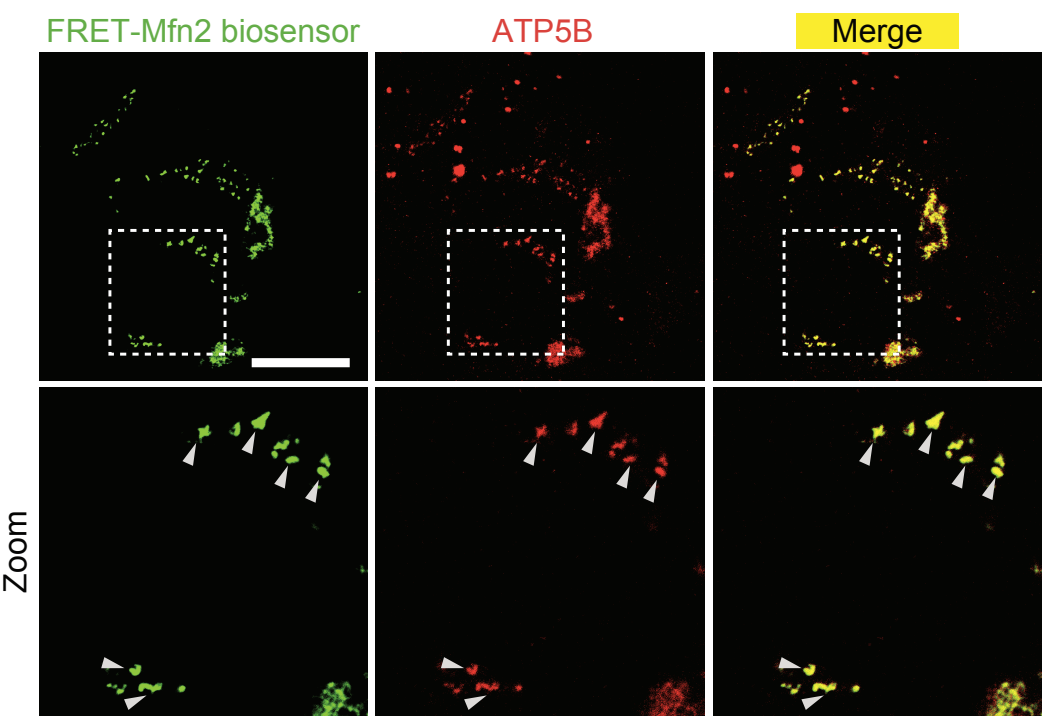
497 Figure S1 (related to Figures 1 and 2). (A) HR1 and HR2 domains in human Mfn1 and Mfn2

498 shares high sequence homology with mouse and rat Mfn1 and Mfn2. HR1 and HR2 residues

499 used in our pharmacophore hypothesis are highlighted in yellow. (B) FRET-based Mfn2

500 biosensor co-localizes with ATP5B in mitochondria in HEK 293T cells. Scale bar $20 \mu \mathrm{m}$.

501 Zoomed images are $3 x$. 
A
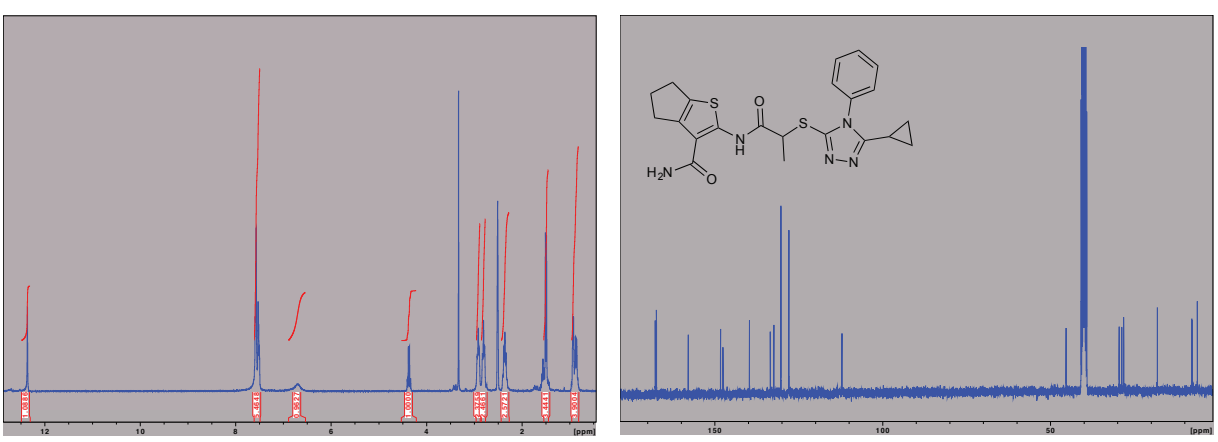

B

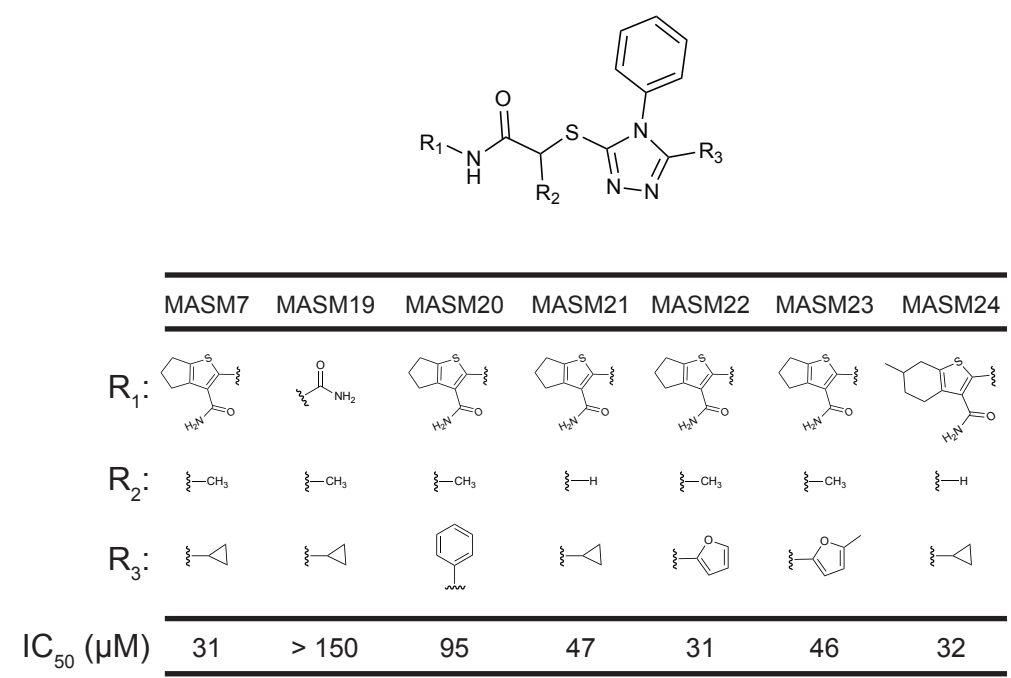

C

D
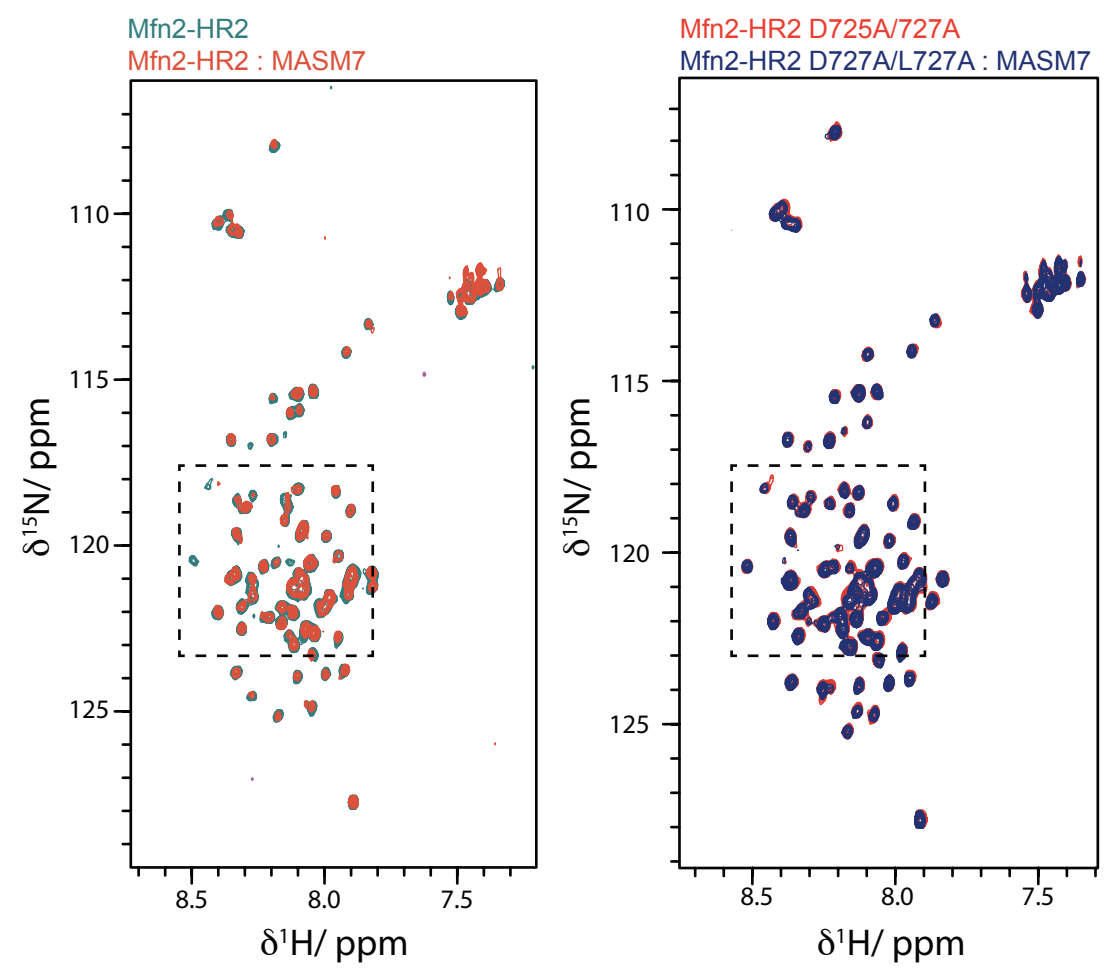
503 Figure S2 (related to Figure 2). (A) Spectral data of MASM7. ${ }^{1} \mathrm{H}$ (left) and ${ }^{13} \mathrm{C}$ (right) NMR.

504 (B) Structure-activity relationship of MASM7 scaffold. Potency of MASMs compounds with

505 variable $\mathrm{R}_{1}, \mathrm{R}_{2}, \mathrm{R}_{3}$ groups were evaluated using WT FRET-based biosensor. HEK 293T cells

506 were treated with MASMs for $2 \mathrm{~h}$. (C) Comparison of the ${ }^{1} \mathrm{H}_{-}{ }^{15} \mathrm{~N}$ HSQC spectra of Mfn2-HR2

$507(50 \mu \mathrm{M})$ with (red cross-peaks) and without (green cross-peaks) MASM7 (200 $\mu \mathrm{M})$. Zoomed

508 region as shown in Figure 2E is highlighted in black box showing overlaid spectra of Mfn2-HR2

509 with and without MASM7 that demonstrates examples of the observed line broadening and

510 chemical shifts upon addition of MASM7 as highlighted with blue boxes. (D) Comparison of the

$511{ }^{1} \mathrm{H}^{15}{ }^{15}$ HSQC spectra of Mfn2-HR2 D725A/L727A mutant (50 $\left.\mu \mathrm{M}\right)$ with (blue cross-peaks) and

512 without (red cross-peaks) MASM7 $(200 \mu \mathrm{M})$. Zoomed region as shown in Figure $2 \mathrm{~F}$ is

513 highlighted in black box showing overlaid spectra of Mfn2-HR2 D725A/L727A mutant with and

514 without MASM7 that demonstrates loss of effects of MASM7 on broadening and chemical 515 shifts. 